\title{
Health disparities and inequalities: a study of migrant workers in Shenzhen, China
}

\author{
Ki Fung Kelvin LAM ${ }^{1}$; Janice Mary JOHNSTON ${ }^{1}$ \\ ${ }^{1}$ School of Public Health, Li Ka Shing Faculty of Medicine, University of Hong \\ Kong
}

\begin{abstract}
Internal migration has induced a profound shift on social structure across China, but the effects on health for migrants remain scarcely reported. In this study we set out to investigate the disparities in health and the potential healthcare inequalities for migrant workers in Shenzhen.
\end{abstract}

Keywords: Migrant, China, Healthcare, Utilisation, Inequality

\section{Introduction}

Rural-to-urban migrant workers have been the backbone of mainland China's prevailing growth and constitutes up to $85 \%$ of the population in special economic zones like Shenzhen city. Migration and rapid urbanization may alter the social, psychological or behavioral risk factors on health and in turn impact health policy and provision of healthcare in the city. In the concurrent background of healthcare reform in China, this study investigates whether migrants suffer from health disparities and inequalities in access to care.

\section{Methods}

Two cross-sectional studies were conducted in 2010 and 2013 to elicit the impact of the recent health reforms on healthcare utilization. A validated semi-structured telephone questionnaire was used to assess the demographic, socio-economic status, self-rated health, mental health status (Centre for Epidemiological Studies Depression scale (CES-D)), behavioural risk factors, health insurance coverage, pattern of healthcare service utilisation and out-of-pocket (OOP) payments of respondents. Descriptive statistics 
and multivariate regression analysis were used.

\section{Results}

The sample $(\mathrm{N} 1=1,543 \quad \&$ $\mathrm{N} 2=1,533)$ was representative of the Shenzhen census figures with satisfactory effect sizes. Migrants were, in general, younger, female, single, have lower levels of education, more likely to be employed and be current smokers (OR $1=1.32 ; \quad$ CI $1.04,1.67 \quad$ \& OR2 $=1.94$; CI $1.50,2.51)$ when compared with registered residents. They were also more likely to have a CES-D score of 16 or above, which is highly suggestive of having depression, $(\mathrm{OR}=1.81$; $\mathrm{CI}$ 1.18,2.76), and report fewer chronic health conditions $(\mathrm{OR} 1=0.48$; CI $0.34,0.67 \quad \& \quad$ OR2 $=0.59 ; \quad \mathrm{CI}$ 0.44,0.79). Though health insurance coverage rose, $35 \%$ of migrants remain uninsured. After adjustments for confounding, stratified regression models show that migrants, particularly those who were uninsured, unemployed and depressed, were less likely to have utilized healthcare services (both in-patient and out-patient). Migrants were consistently more likely to make OOP payments despite the increase in insurance coverage. The average OOP payment increased (out-patient services: RMB271.28
RMB304.76 \& in-patient services: RMB3429.57 > RMB4611.33).

\section{Conclusion}

The findings of this study reflect the vulnerabilities of being migrant in which their poorer health status is coupled with less access to healthcare, potentially contributing to the Inverse care law. Whilst health reforms have increased health insurance coverage, moral hazard as well as little improvements to OOP payments was observed. The evidence suggests that migration has contributed to health inequity and inequality in China, and recent reform efforts may require further improvements in the provision of health services to address these issues.

\section{References}

[1] Lam KK, Johnston JM. Health insurance and healthcare utilisation for Shenzhen residen ts: a tale of registrants and migrant s? BMC Public Health. 2012 Oct 12;12:86 